\title{
TRIAXIAL SYMMETRIC ROBOTS: STATE OF THE ART AND TRENDS
}

\author{
Viorel Gheorghe ${ }^{1}$, Daniel Comeagă ${ }^{2}$, Despina Duminică ${ }^{3}$, Adrian Cartal $^{4}$ \\ 1,2,3,4 University POLITEHNICA of Bucharest \\ viorel.gheorghe@upb.ro,comeagadaniel@yahoo.com,despina.duminica@upb.ro \\ adrian.cartal@upb.ro
}

\begin{abstract}
Mobile robots currently attract huge interest due to their extraordinary palette of applications, from intelligent toys to complex industrial uses.

Different categories of applications have brought the need to develop a wide variety of original locomotion systems, depending on the particular task that needs to be executed. Therefore, several aspects must be considered: the locomotion type, the environment in which the movement is performed, the dynamic requirements (i.e. speed, acceleration), sensor endowment, and safety measures to ensure both the preservation of the robot and of the surrounding life forms.

A new trend, increasingly explored lately, consists of using triaxial symmetric robots with rolling and/or jumping locomotion capabilities.

Triaxial symmetric robots can be identified as a typology of robots with geometrical symmetry features on all three axes, showing multiple advantages, like: fast recovery after a collision with an obstacle, no overturning risk, the ability of moving in various directions without needing additional movements when switching positions.

These characteristics recommend the implementation of the above mentioned robotic structures in situations when the need for multi-modal locomotion concepts arises, the risk of hitting obstacles is unavoidable, high resilience is required, landform transition environment (i.e. shallow waters, swamps), and for surveillance and reconnaissance tasks.

The development perspectives in the area are practically endless, therefore the article performs a summary of the state of art in these practical locomotion solutions and proposes new research directions in the same area.
\end{abstract}

Keywords: triaxial, symmetric, mobile robots, rolling, spherical, radial legs.

\section{Introduction}

Over the past two decades a new typology of mobile robots, that presents high geometrical symmetry have catch the interest of researchers in the field.

Triaxial symmetric robots (TSR) comprises robotic structures with a high degree of geometrical symmetry features on all three axes. Due to their external form and driving mechanisms present a series of advantages: fast recovery after a collision with an obstacle, no overturning risk and the ability of moving in various directions without needing additional movements when switching positions.

At the beginning, the sphere was the first chosen form for the triaxial symmetric robots, later on another approaches have been studied [1], [2] and [3].

The vast majority of TSRs use rolling as main locomotion method, while some presents also jumping, and even flight/diving abilities.

In order to create self-locomotion a plethora of designs are proposed and only few reach the prototype phase. All of these solutions can be categorise in two main sections for triaxial symmetric robots: rigid shell robots and deformable shell robots. While in the first section the propulsion principles resides in shifting the centre of mass of the robotic structure and/or angular momentum conservation, the second section relies mainly on directed shell deformation movement due to smart materials actuation.

The following parts of this paper include an overview of the locomotion principles and other important characteristics of rigid shell TSR robots (part II), continued by deformable TSR robots (part III). At the end some conclusions and trends in design and development of triaxial symmetric robots are revealed (part IV).

\section{Rigid Shell Triaxial Symmetric Robots}

When triaxial symmetric robots have made their appearance, in 1990s, one of their main characteristics were the spherical shape and hard external protective shell.

One main typology of non-deformable TSRs use sphere like shells, which harbours inside the 
structure the driving unit of the robot. Beside this, another typology have outer shell actuation mechanisms.

\section{A.Inner Shell Actuated Robots}

Vast majority of TSRs use as locomotion principle the displacement of the centre of mass away from its equilibrium state, less frequently is used angular momentum conservation and some other concepts.

\section{a)Internal Rolling Structures}

An often used principle is usage of actuated rolling structures based on wheels/spheres, which in contact with the internal shell surface shifts the centre of mass of the entire robot into the desired movement direction. The internal drive unit (IDU) is kept in contact with the shell due to its own weight or different types of elastic mechanisms.

One of the first TSRs ever studied is Sphericle [4]. Its principle was based on moving an internal mass inside the robot with the help of a two-wheel differential drive system (Figure 1).

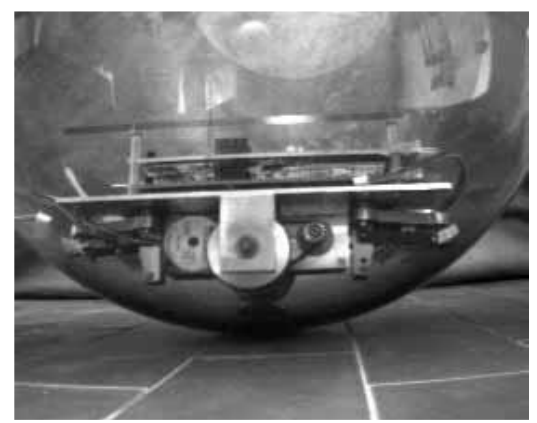

Figure 1: Robot "Sphericle"

The moving platform is a minicar kept in contact with the sphere, by its own weight, and is stabilised with the help of two suspension arms, minimising in this way the wheel slippage.

There are more recent prototypes that use differential drive wheels [5]. In this case, with the help of rapid prototyping techniques, a spherical robot of almost $60 \mathrm{~mm}$ diameter, that weights $55 \mathrm{~g}$ has been developed (Figure 2).

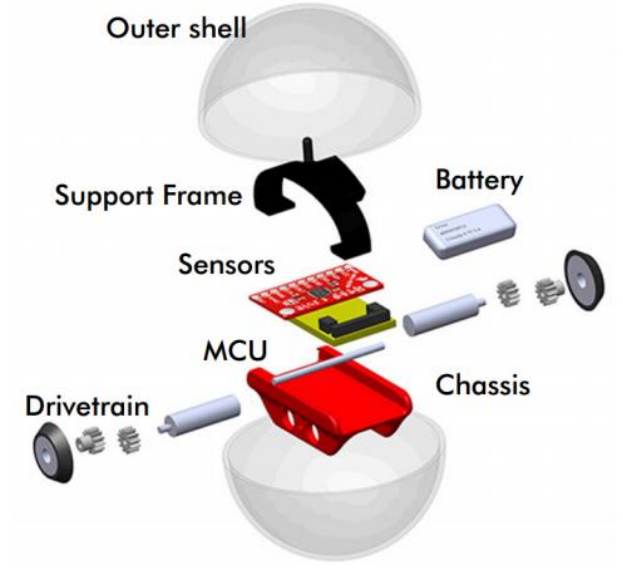

Figure 2: Exploded view of the miniature robot
Its control techniques involves a proportional derivative (PD) controller and is capable of reaching a top speed of $0.16 \mathrm{~m} / \mathrm{s}$.

Another used approach is based on a three omniwheel platform housed into a spherical shell [6]. The obtained robotic structure presents holonomic abilities due to the symmetrical arrangement of the wheels and of course of the abilities of the omniwheels; active rolling movement and passively sideways movement (Figure 3).

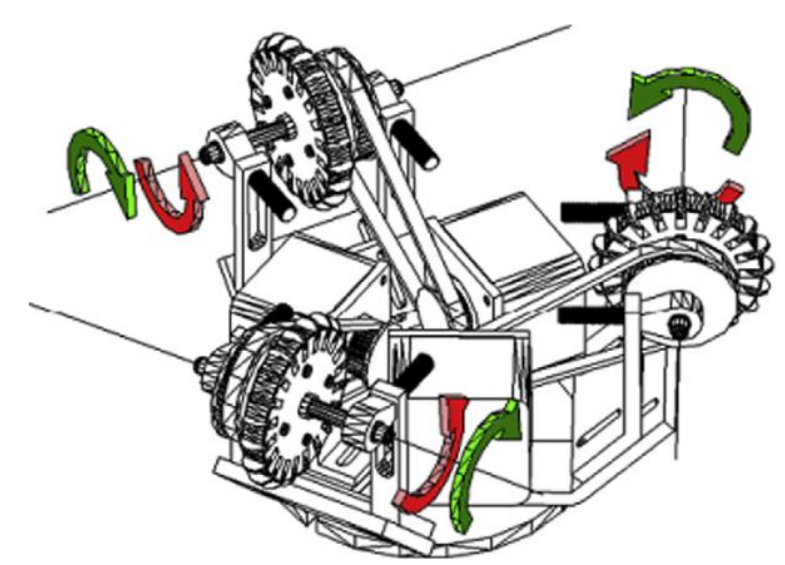

Figure 3: Internal drive unit with omni-wheels

The tests done with this prototype with an external spherical shell of $250 \mathrm{~mm}$ diameter obtained a linear average speed of $0.8 \mathrm{~m} / \mathrm{s}$ while the real trajectory compared is close to the simulated one.

Mobility of the TSRs can be achieved by using an IDU formed by a single wheel that can move the internal base of the robot inside a hallow sphere, disturbing the system equilibrium, and thus achieving the desired rolling of the entire structure. [7]. In this case, all the necessary electronics (sensors, drives, motors, batteries) are mounted on the IDU. On the same structure can be found a steerable driving wheel kept in contact with the hallow spherical case by using an elastic spoke mechanism based on a balance wheel and a spring (Figure 4).

The iconic Ball Robot built at the end of the 1990s had a $250 \mathrm{~mm}$ diameter, a weight of $3.2 \mathrm{~kg}$ and an untether operating mode.

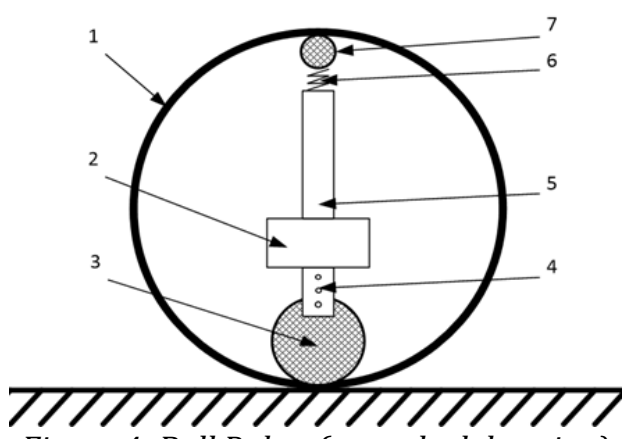

Figure 4: Ball Robot (reworked drawing)

1. case, 2. IDU base, 3. driving wheel, 4. steering axis, 5 . supporting axis, 6. spring, 7. balance wheel 
Internal rolling driving units can be in some cases a driven ball actuated by two orthogonally-mounted rollers that is in close contact with its spherical shell, thus obtaining an omnidirectional robot [8].

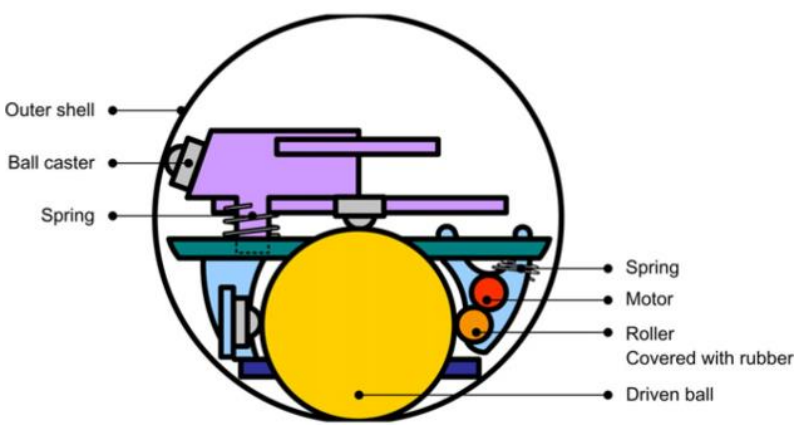

Figure 5: OmniQiu: internal construction

The robot is capable of singularity-free omnidirectional locomotion using the direct-driving method due to the internal actuated ball. The design is a feasible one, although it has some lateral deviation and oscillations when traveling straight lines.

\section{b) Pendulum Type Drives}

Due to its simplicity and somehow easier control is one of the preferred design types of TSRs. In order to shift the position of the centre of mass a pendulum with two degrees of freedom can be used The design usually has a pendulum connected to a main axle actuated by two perpendicular motors which moves the centre of mass in the desired direction.

Groundbot, one of the most robust TSRs has this locomotion principle [9]. Its performance for a diameter of $600 \mathrm{~mm}$ is a top linear speed of $3 \mathrm{~m} / \mathrm{s}$ and is capable to handle rough outdoor terrain: snow, mud, sand or water (Figure 6).

[line]

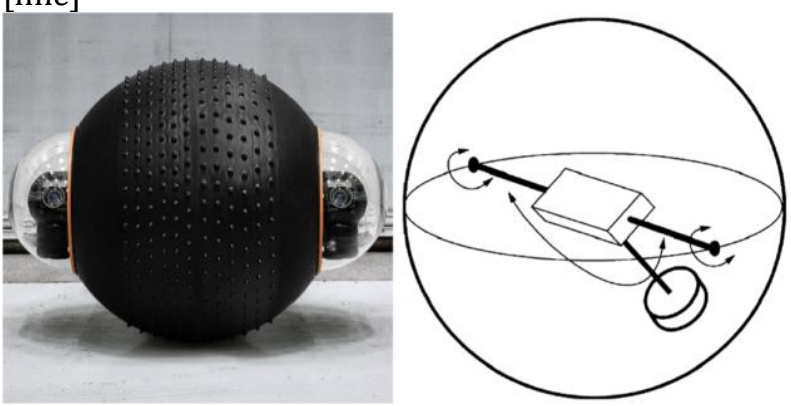

Figure 6: Groundbot (left) and its pendulum locomotion based principle (right)

In order to overcome some of the problems of classical solution of spherical robots the robot KisBot is capable of two driving modes: rolling and wheeling [10]. This is achieved by having a spherical outer shell and two extending arms.

The mechanics of the robot comprises a wheelshape central body mounted between two rotating hemi spheres, each of them containing an arm (Figure 7).

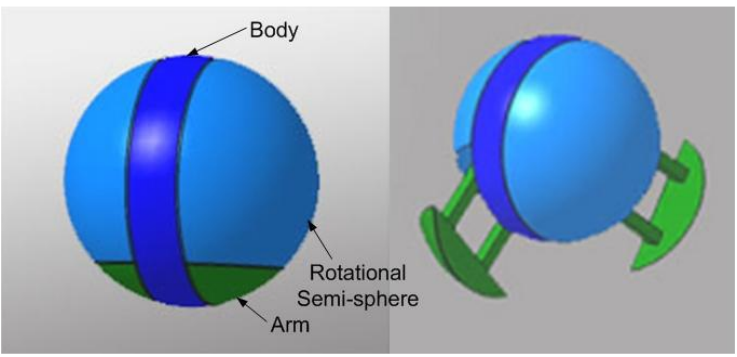

Figure 7: CAD model of KisBot

In order to roll the robot needs to have the legs retracted and the hemispherical parts to act as double pendulum actuation mechanism. If the arm are extended the robot movement will be similar to a wheel robot, having the arms as ground support and the central body as the wheel.

A variant of this propulsion solution is presented in [11], where two independent pendulums are used (Figure 8). An interesting double pendulum driving unit is described in [12] and even a four pendulum solution [13].

[line]

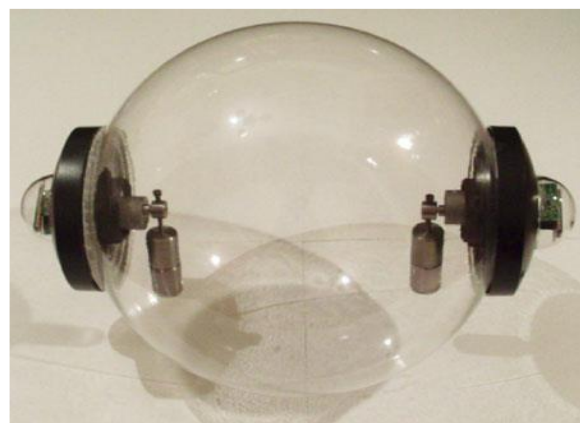

Figure 8: Robot actuated by double pendulum

c)_Angular Momentum Conservation Drives Another approach in moving a TSR is using the principle of conservation of angular momentum.

One implementation solution is to place two orthogonal actuated metal rotor inside a spherical enclosure [14]. In order to achieve the coincidence of the centre of mass with the geometrical centre of the sphere two dead weights have been placed diametrically opposite with the active units (Figure 9). The obtained robotic structure has an outside diameter of roughly $600 \mathrm{~mm}$ and a weight of $3.4 \mathrm{~kg}$.

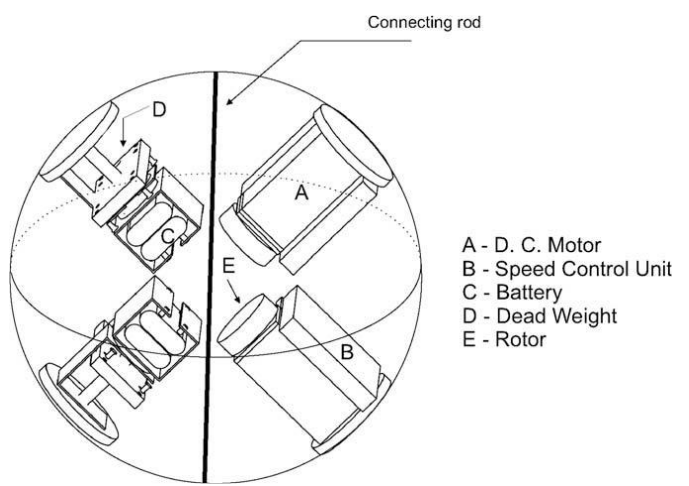

Figure 9: Robot actuated by conservation of angular momentum 
There have been also studies regarding a concept that involves three orthogonal actuated rotors [15]. Thus, is created an inner drive mechanism based on angular momentum conservation (Figure 10).

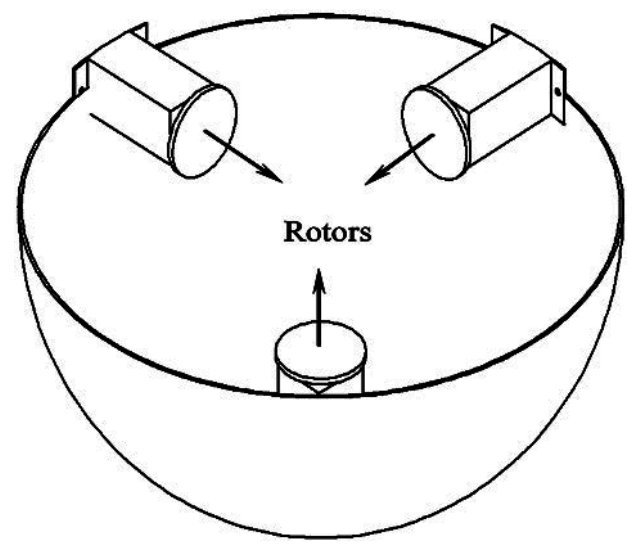

Figure 10: Robot concept with three orthogonal rotors

\section{d)Distribute Mass Drives}

One of the earliest spherical (triaxial robots) concept had a propulsion mechanism based on distribution of internal discrete weights.

Spherobot had four internal radial spokes fixed inside the sphere like a virtual tetrahedron and each one of them having a weight that can move along the spoke (Figure 11). Controlling the position, speed and the acceleration of the four weights along their spokes ultimately determines the position, speeds and the acceleration of the whole robot. The concept also mentions a retractable camera and telescopic limbs that can be deployed when robot is at rest ad retracted when the robots moves [16].

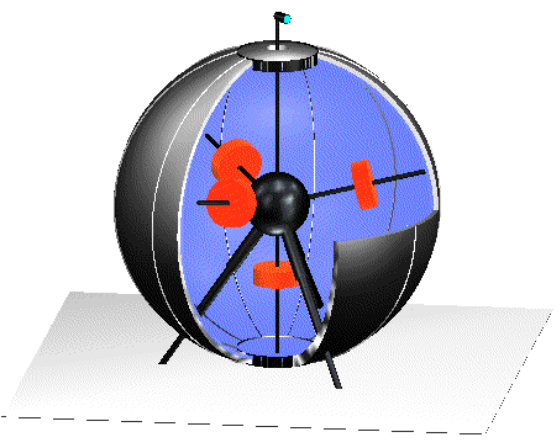

Figure 11: Spherobot

A hardware implementation of a similar robotic structure robot is embodied in an acrylic transparent outer shell of $4 \mathrm{~mm}$ thickness, with an inner radius of $360 \mathrm{~mm}$ protects the internal components and ensures the rolling surface [17].

The centre of mass is shifted with the help of four power screwed spokes connected with one end on the outer shell and to the other to an internal tetrahedral inner shaped core. The actuation of the weights that ensures locomotion is performed by stepper motors mounted directly to the spokes by a screw-nut mechanism (Figure 12).

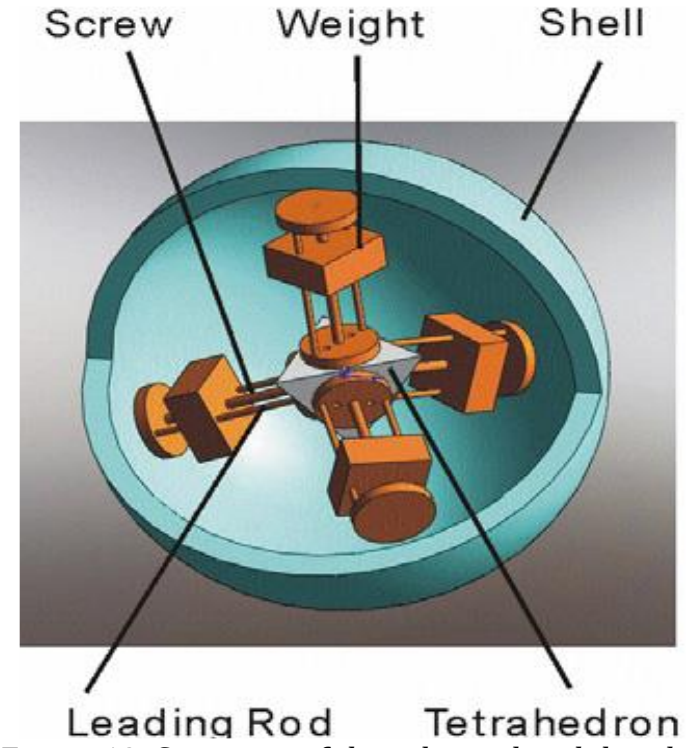

Figure 12: Structure of the spherical mobile robot

\section{e) Other Locomotion Concepts}

A robotic concept that use a fluid multi-driven closed system is "RollRoller" [18], that has 4 degree of freedom (forward/backward, lateral, orientation and jumping). The structure has a spherical rolling protective shell that harbours a series of circular and elliptic pipes, mounted in two plane perpendicular to each other (Figure 13).

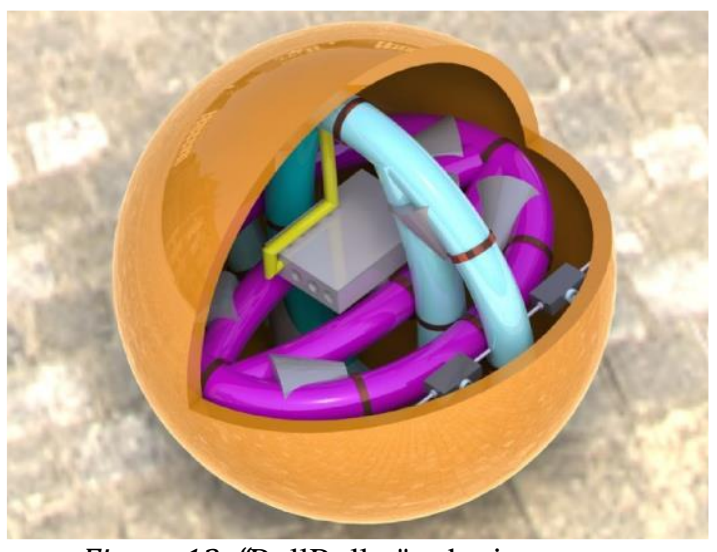

Figure 13: "RollRoller" robotic concept

Two high density metallic spherical cores are put into the perpendicular pipes, one that will generate the movement trust and the one for controlling the direction of the robot. The cores are moved using high density flow fluid created by multi-function linear electrical actuators linked to a base hydraulic cylinder.

Micro aerial terrestrial robots capable to fly through the air or roll on the ground have also been developed [19]. Basically, is a micro-quadcopter mounted in a lightweight spherical exoskeleton that can rotate about the quadcopter (Figure 14). 


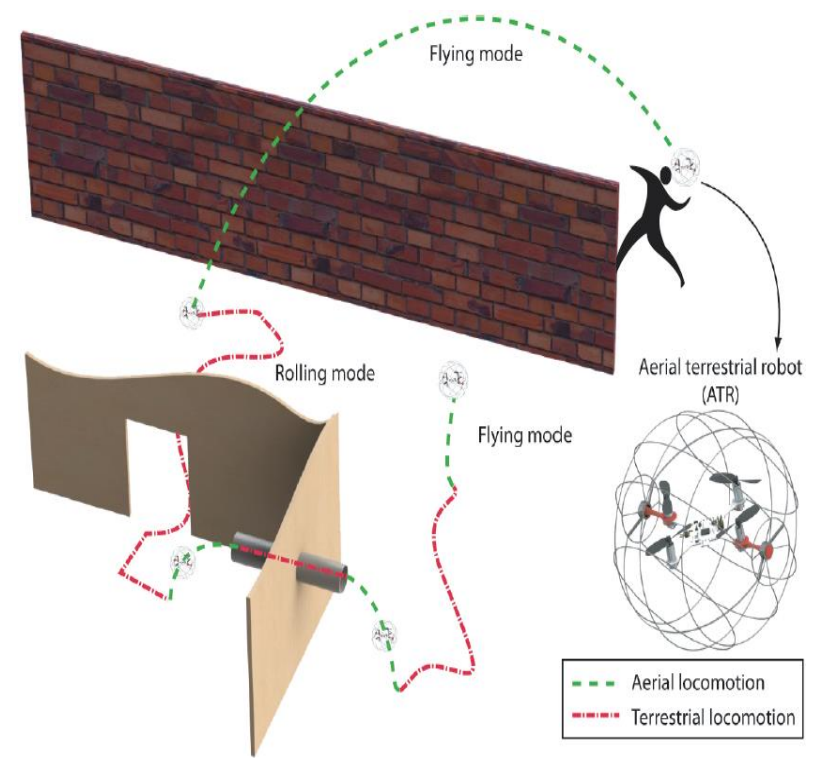

Figure 14: Rolling and flying aerial terrestrial robot

The hover-capable robot has the external exoskeleton structure coupled through two rotating supporting axles that ensures the rolling capability about the quadcopter's central axis. The outer shell has two flat surfaces right about the end of each axle and creates a resting surface. The experimental results seem promising taking in account the distance travelled with only one battery charge. Thus, the micro aerial terrestrial robot is 2.63 more efficient in its rolling stage compared to a traditional fly only robot, and in flying stage the efficiency is 0.61 .

When cluttered environments have to be passed through and is difficult to endow a robot with complex sensing systems and 3D mapping strategies for obstacle avoidance, a solution might be a robotic structure with high degree of resilience.

This scenario is even harder to implement if the main locomotion form of the robot has to be flight. Gim-Ball is a flying robot that can cope in these situation, having a protective external mesh that can rotate passively thanks to a 3-axis gimbal system, helping the inner flying core to maintain its stability [20].

The protective mesh has a spherical polyhedron shape and decreases as little as possible the aerodynamics of the flying robotic platform that can hover and can have a slow lateral flight (Figure 15).

In order to reduce the weight is used only one thruster: a co-axial motor with two propellers that ensures a good lift per area ratio. Direction is kept by using two actuated control surfaces (roll \& pitch) and by differential actuation of the coaxial motor (yaw).

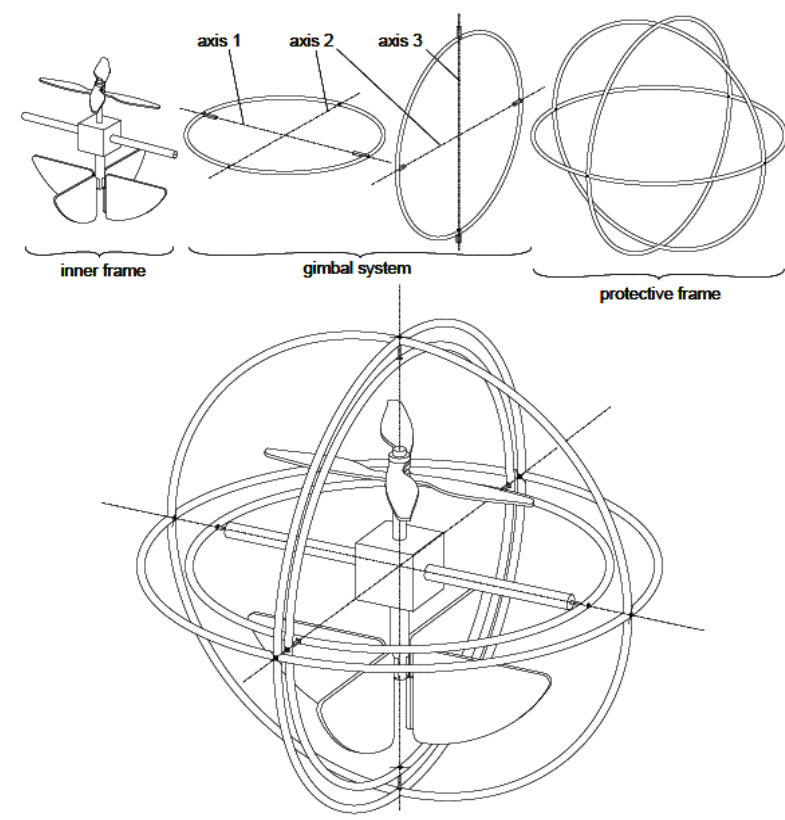

Figure 15: Gim-Ball flying robot

The symmetrical shaped robots have been design for underwater tasks also, gaining with this shape increased shock resistance and flexibility [21].

The SUR-II robot was design to be deployed by an aircraft or from a ship, directly into the water, and to ensure a high shock resistance a spherical shell shape was chosen.

The robotic structure has a cylindrical pressure vessel and two hemispheric hulls joined together joined together in such a way in which provides an adjustable centre of buoyancy (Figure 16).

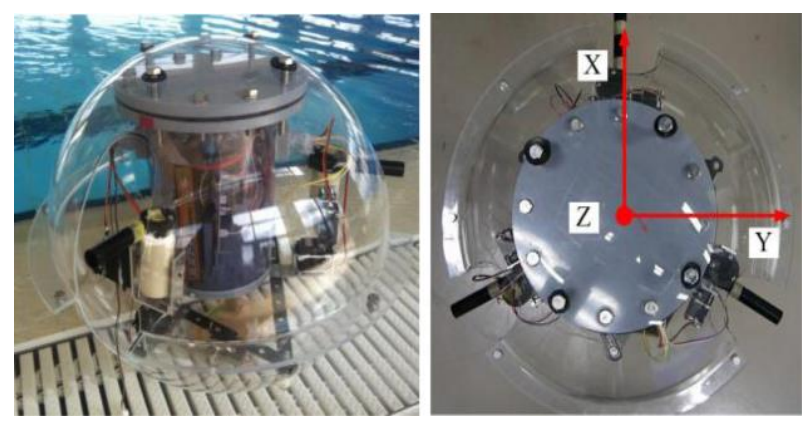

Figure 16: Spherical underwater robot (SUR-II)

The hemispherical hulls have several openings for the propulsion composed of three vectored water-jet thrusters and a triangular support. The propulsion system is mounted in the lower hemisphere, while the electrical system resides into the pressure vessel.

The vectored water-jet thrusters permit 4degrees of-freedom and because is installed within the hull, prevents them from external impacts, reducing their influence of the hydrodynamic characteristics of the robot and finally makes the construction more compact. 


\section{B.Outer Shell Actuated Robots}

Another types of triaxial symmetric robots are those with outer shell driving capabilities, due to radial disposed legs or some other appendages.

In [22] is presented a concept of rolling robot with twelve extendable legs that are mounted on a dodecahedral nucleus (Figure 17). The locomotion is achieved by shifting the robot mass centre position, by retracting and extending rapidly one or more legs.

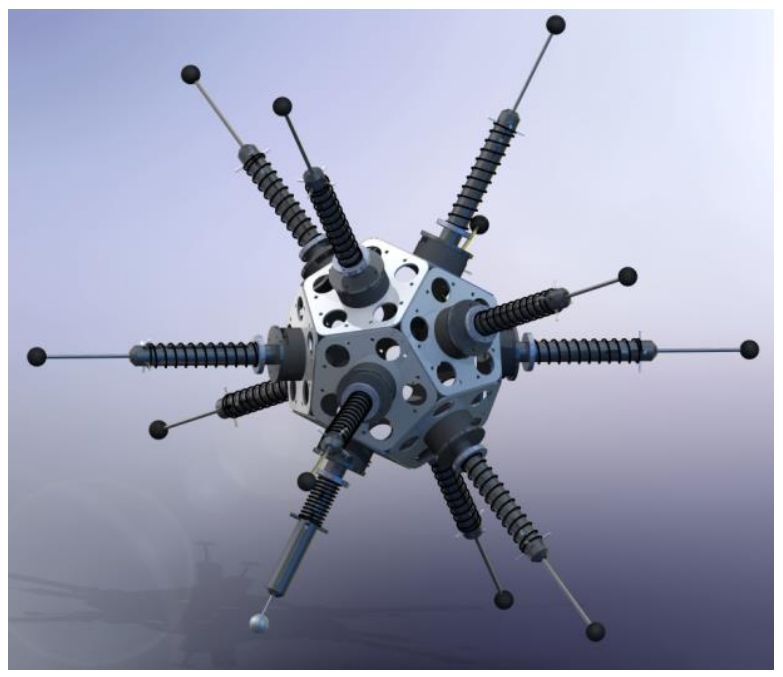

Figure 17: Dodecahedral Robot

Usually the robot stands on three legs, one of them being at one time the driven leg, while the other two are used just like support legs.

Figure 18 presents the robot movement that implies a series of phases in which the legs are retracted/extended, each leg having a single degree of freedom.

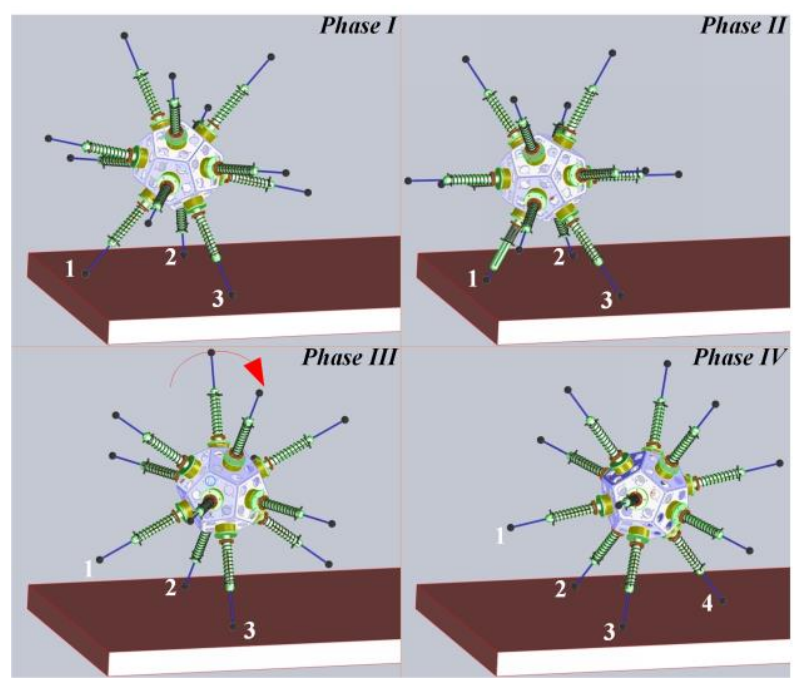

Figure 18: Locomotion phases

Due to its construction the trajectory of the robot will describe a discrete zigzag trajectory.
A structure that derives from a symmetrical geometric body is the robot "Spike" [23]. The central structure is a cube having legs attached on each of its faces (Figure 19). Every leg has two degrees of freedom that helps the robot to move by consecutive tumbles. Since every step (tumble) can be executed only in three directions in case of a straight desired trajectory the actual trajectory will have a trapezoidal shape around the targeted proposed plan.

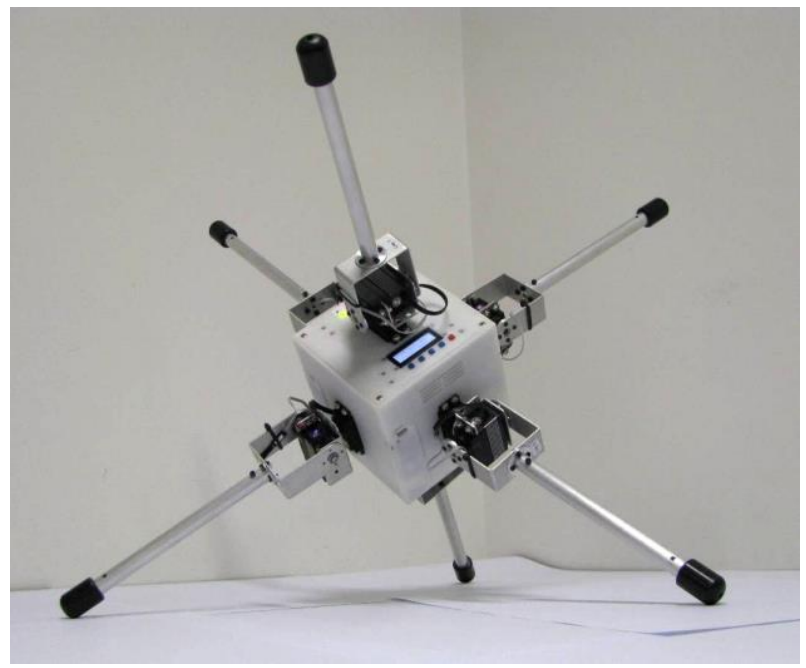

Figure 19: The "Spike" Robot

A triaxial robot concept comprise a pendulum-based spherical robot circumscribed into a bigger spherical shell [24]. The external shell is similar with the sea urchin exoskeleton due to the fact it contains retractable telescopic legs uniformly dispose over the surface and gives the robot the ability to overcome greater obstacles (Figure 20). [line]

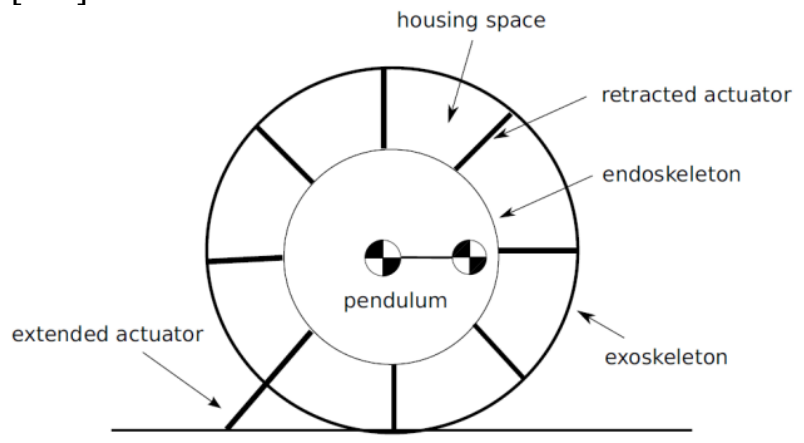

Figure 20: Sea-urchin Robot Concept

Robotic All-Terrain Surveyor (RATS) is a robotic prototype that has a spheroidal body, the size of a soccer ball with 12 legs symmetrically distributed around its surface (Figure 21).

This design that can emulate a variety of locomotion modes, like hopping, tipping and rolling. Each leg consist is a single DOF pneumatic linear actuator attached to the central core of the robotic 
structure - a regular dodecahedron, that contains a high-pressure nitrogen gas tank [25].

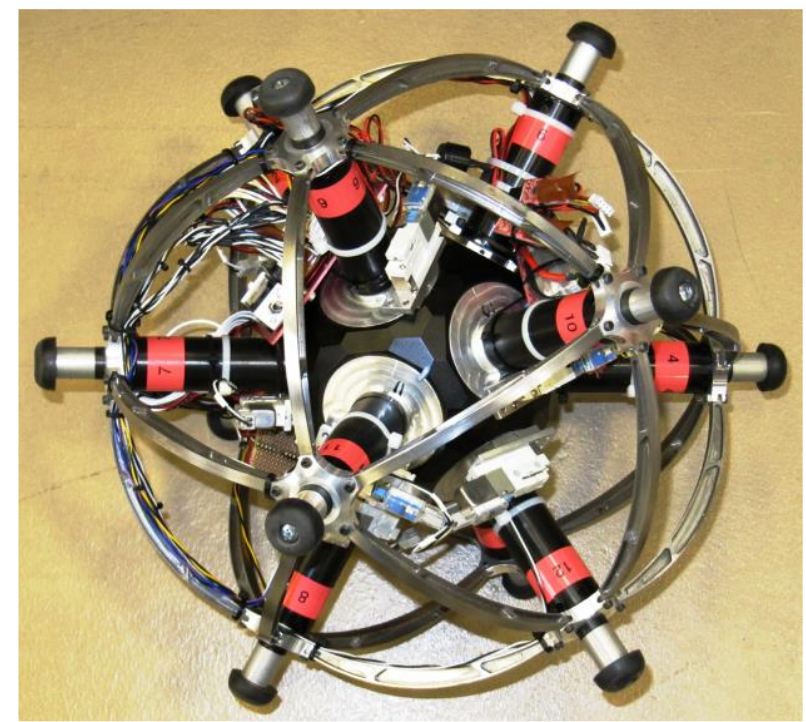

Figure 21: RATS: a 12-legged pneumatic robot

Another solution of legged symmetrical structure comprises a spherical rolling shell and four legs that have oscillatory movement (Figure 22). Actuating the legs in a predefined pattern the motion is achieved due to the centre of mass movement, reducing the usual slippage of the normal spherical robots [26].
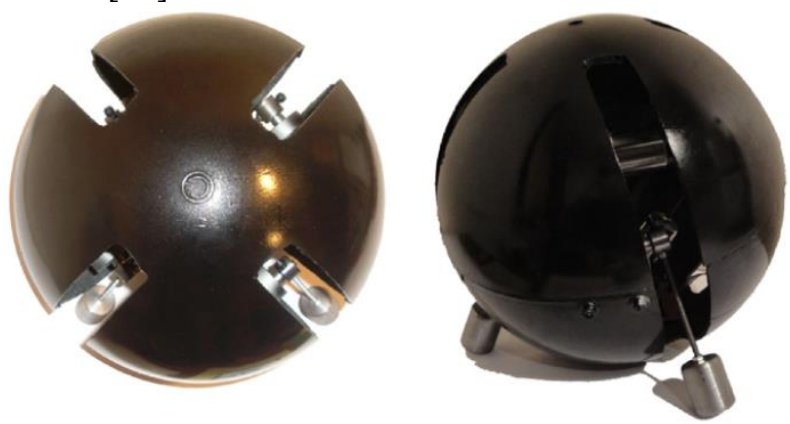

Figure 22: Quadruped spherical mobile robot

\section{Deformable Shell Triaxial Symmetric Robots}

In this category can be found robots with soft or flexible multibody design, along with those that poses a rigid multibody one.

Jollbot is a robot that realize motion through a combination of jumping and rolling [27].

Its outer shell is constructed from metal elastic semi-circular hoops that can are tensioned with the help of an internal mechanism in order to store the energy used for jumping. By rapidly releasing the energy stored in the elastic hoops in a selected direction the robot can jump and roll.
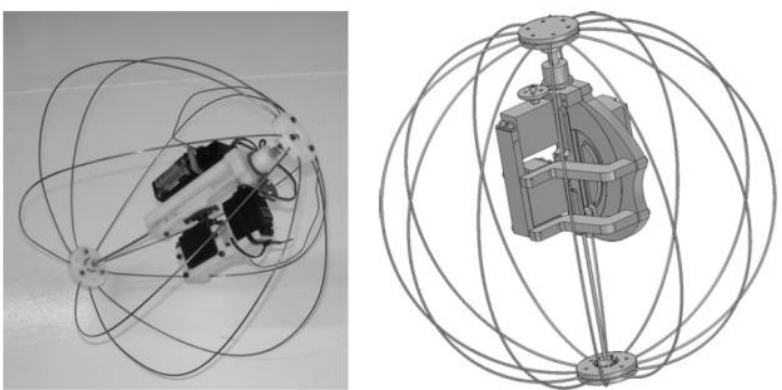

Figure 23: Jollbot (prototype and CAD model)

Shape shifting robots can also be constructed with unconventional actuators: shape memory alloy [28]. Koharu is a family of robots that can crawl/roll, and jump and have a spatially symmetric form (Figure 24).

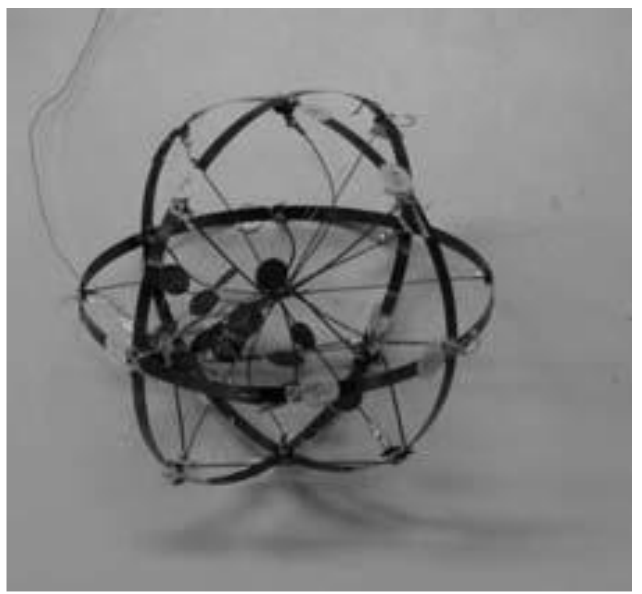

Figure 24: Koharo: spherical soft robot

The prototypes have several spring steel flat rings at the exterior and around 20 shape memory wires that connects the steel rings with the core of the robot. There are versions endowed with electronic control and communication modules and versions with external control. Tests made with the lighter version (the control and power modules were outside the robot) shown that can be achieved heights double of its diameter $(90 \mathrm{~mm})$, while having a mass of only $5 \mathrm{~g}$.

Deformable shell TSRs have been constructed also with dielectric elastomer actuators (DEA) [29]. The deformable structure has the external surface made from sectors of elastomers, which have the ability to change their shape by a given imposed electrical field, thus modifying the centre of gravity causing the structure to roll.

The rover has a 3D printed rigid internal frame with four ribs, covered by dielectric elastomers, separated in four different sectors that can be activated independently (Figure 25). In order to function the DEA has to be prestreched, and in this particular case this is achieved by pumping air into the structure, thus giving it a spherical form. 
The tested prototype had a linear velocity of 43 $\mathrm{mm} / \mathrm{s}$ for an external radius equal to $54 \mathrm{~mm}$.

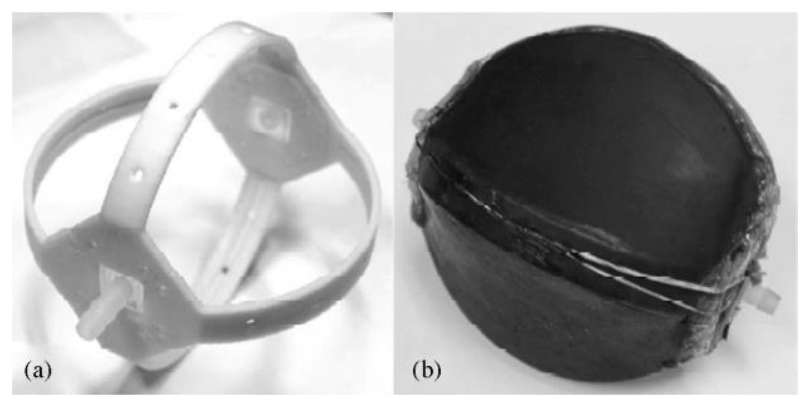

Figure 25: Deformable Spherical Rover a) internal frame. b) robot prototype non inflated

An interesting inflatable TSRs is presented in [30].

The tested prototype comprises numerous individually inflatable rubber bladders of spherical pentagonal/hexagonal shape, much like a soccer ball

Locomotion is achieved by inflating the rubber bladders in a selected sequence that produces the change of the centre of gravity and thus producing the rolling of the robot. Each elastomer actuators are filled with air using a pneumatic valve that is controlled opto-electronically through infrared signals (Figure 26).

In order to reduce the computational power needed to control the robot, an opto-mechanical system is used to determine the direction of the gravity vector and which bladder should be filled in order to respect the director of travel, by illuminating the proper light sensitive pneumatic valve.

For a prototype of $356 \mathrm{~mm}$ and a mass of $9.1 \mathrm{~kg}$, a linear speed of $76 \mathrm{~mm} / \mathrm{s}$ was obtained, working in tethered operation mode having plugged a pneumatic hose with a pressure of 2.1 bar.

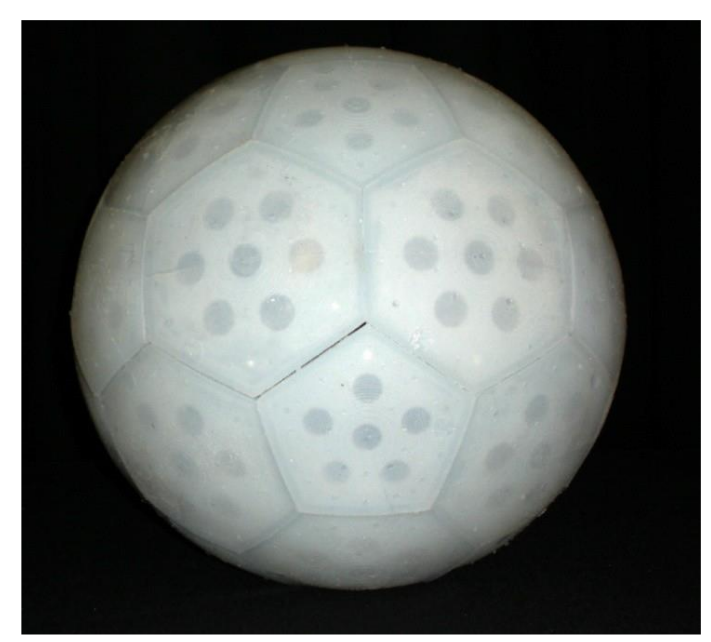

Figure 26: Pneumatic rolling robot

One interesting concept that ensures an easier and more energy efficient steering mode due to control of the outer shell deformation is presented in [31].
The structure consist in a central nondeformable module, and two lateral deformable hemispheres. The central module contains a pendulum type drive unit and seals from the environment the most important parts of the robot. The lateral deformable parts contain of rigid shell parts actuated through a slider crank mechanism (

Figure 27).

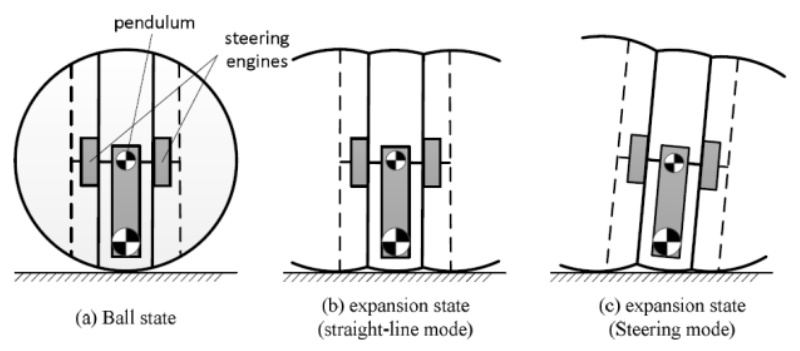

Figure 27: Deformable robot

A soft spherical shell robot with a predefined rolling axis can be found in [32]. The actuation is based on a pendulum type mechanism, having two electrical inphase motors disposed symmetrically on the rolling axis with their shells fixed on the bracket, while the armatures of them are on the soft shell of the spherical robot to provide drive moment of force ((Figure 28).

In order to steer the pendulum is used one motor, which ensures a degree of freedom of the load. The elasticity and plasticity of soft shell is represented by some uniform springs and used to create the kinetic model deduced from Newtonian mechanics principles.

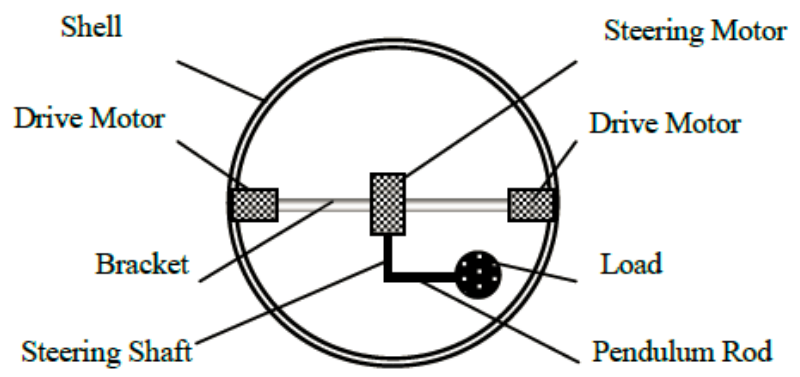

Figure 28: Soft shell robot-inner mechanisms

\section{Conclusions}

Triaxial symmetric robots can be a feasible solution for surveillance and reconnaissance tasks that might include unavoidable risk of hitting obstacles, high resilience requirements, landform transition environment (i.e. shallow waters, swamps).

Regarding shape, there are situations in which outer shell limbs/legs can be used in order to prevent the robot of being stuck.

Energy source type, probably is best to be electrical, since is often more easily to produce on site (solar panels, dedicated charge spot, etc.). 
Probably in the next years designs based on smart materials actuation will appear, especially in the deformable TSRs section, but these actuators have usually an unpractical power requirements, which makes them less desirable.

For practical reasons a relatively stable rotation axis will be preferable, because permits endowment of the robot with more easily usable sensors.

In order for the robot to be energy wise and capable of overcoming greater obstacles, combinations of the presented locomotion principles can be implemented.

\section{Acknowledgements}

This work has been funded by University POLITEHNICA of Bucharest, through the "Excellence Research Grants" Program, UPB - GEX. Identifier: UPB-EXCELENȚĂ-2016, “Conceperea, dezvoltarea și realizarea unei platforme de test tip robot simetric triaxial" - "Design, Development and Implementation of a Triaxial Symmetric Robot Test Platform", Nr. 37 / 2016.

\section{References}

[1] V. Crossley, "A literature review on the design of spherical rolling robots," Pittsburgh, PA, pp. 1-6, 2006.

[2] J. Suomela and T. Ylikorpi, "Ball-shaped robots: An historical overview and recent development at TKK," Field and Service Robots, STAR 25, pp. 343-354, 2006.

[3] R. Chase and A. Pandya, "A Review of Active Mechanical Driving Principles of Spherical Robots," Robotics, vol. 1, no. 1, pp. 3-23, 2012.

[4] A. Bicchi, A. Balluchi, D. Prattichizzo, and A. Gorelli, "Introducing the Sphericle: an experimental testbed for research and teaching in nonholonomy," in Proc. IEEE Int. Conf. on Robotics and Automation, 1997.

[5] X. Niu, A. P. Suherlan, G. S. Soh, S. Foong, K. Wood, and K. Otto, "Mechanical development and control of a miniature nonholonomic spherical rolling robot," in 2014 13th International Conference on Control Automation Robotics and Vision, ICARCV 2014, 1997, pp. 1923-1928.

[6] H. Ghariblu, "A new mobile ball robot-dynamic modeling and simulation," Appl. Math. Model., vol. 39, no. 10-11, pp. 3103-3115, 2015.

[7] A. Halme, J. Suomela, T. Schönberg, and Y. Wang, "A Spherical Mobile Micro-Robot for Scientific Applications," in 4th ESA Workshop on Advanced Space Technologies for Robot Applications, Noordwijk, 1996, The European Space Agency, 1996, p. 3.

[8] W.-H. Chen, C.-P. Chen, T. Jia-Shiuan, J. Yang, and P.-C. Lin, "Design and implementation of an omnidirectional spherical robot," Mech. Mach. Theory, vol. 68, pp. 35-48, 2013.

[9] V. Kaznov and M. Seeman, "Outdoor navigation with a spherical amphibious robot," in IEEE/RSJ 2010 International Conference on Intelligent Robots and Systems, IROS 2010 - Conference Proceedings, 2010, pp. 5113-5118.

[10] Y.-M. Kim, S.-S. Ahn, and Y.-J. Lee, "KisBot: new spherical robot with arms." pp. 63-67, 2010.

[11] S. Mahboubi, M. M. Seyyed Fakhrabadi, and A. Ghanbari, "Design and Implementation of a Novel Spherical Mobile Robot," J. Intell. Robot. Syst., vol. 71, no. 1, pp. 43-64, 2013.

[12] L. Wang and B. Zhao, "Dynamic modeling and control strategy for turning in place motion of a two-coaxial pendulums driven spherical inspector based on stick-slip principle," Mech. Mach. Theory, vol. 83, pp. 69-80, 2015.

[13] B. P. DeJong, E. Karadogan, K. Yelamarthi, and J. Hasbany, "Design and Analysis of a FourPendulum Omnidirectional Spherical Robot," J. Intell. Robot. Syst., vol. 86, no. 1, pp. 3-15, Apr. 2017.

[14] V. A. Joshi, R. N. Banavar, and R. Hippalgaonkar, "Design and analysis of a spherical mobile robot," Mech. Mach. Theory, vol. 45, no. 2, pp. 130-136, 2010.

[15] M. R. Azizi and D. Naderi, "Dynamic modeling and trajectory planning for a mobile spherical robot with a 3Dof inner mechanism," Mech. Mach. Theory, vol. 64, pp. 251-261, 2013.

[16] R. Mukherjee, M. A. Minor, and J. T. Pukrushpan, "Simple motion planning strategies for spherobot: a spherical mobile robot," in Proceedings of the IEEE Conference on Decision and Control, vol. 3, IEEE, 1999, pp. 2132-2137.

[17] W. U. H. C. S. A. N. Q. SANG Shengju ZHAO Jichao, "Modeling and Simulation of a Spherical Mobile Robot," Comput. Sci. Inf. Syst., no. 13, pp. 51-62, 2010. [18] S. A. Tafrishi, S. M. Veres, E. Esmaeilzadeh, and M. M. Svinin, "Dynamical Behavior Investigation and Analysis of Novel Mechanism for Simulated Spherical Robot named 'RollRoller,'” CoRR, vol. abs/1610.0, 2016.

[19] C. J. Dudley, A. C. Woods, and K. K. Leang, "A micro spherical rolling and flying robot," in IEEE International Conference on Intelligent Robots and Systems, 2015, vol. 2015-Decem, pp. 5863-5869. [20] A. Briod, P. Kornatowski, J. C. Zufferey, and D. Floreano, "A collision-resilient flying Robot," J. F. Robot., vol. 31, no. 4, pp. 496509, 2014.

[21] C. Yue, S. Guo, M. Li, Y. Li, H. Hirata, and H. Ishihara, "Mechatronic System and Experiments of a Spherical Underwater Robot: SUR-II," J. Intell. Robot. Syst., vol. 80, no. 2, pp. 325-340, 2015. 
[22] V. Gheorghe, N. Alexandrescu, D. Duminica, and L. A. Cartal, "Rolling robot with radial extending legs," in Proceedings - ISRCS 2010 - 3rd International Symposium on Resilient Control Systems, 2010.

[23] Coyte C., Beckerleg M., Collins J. (2009) "Spike: A Six Legged Cube Style Robot.”, In: Xie M., Xiong Y., Xiong C., Liu H., Hu Z. (eds) Intelligent Robotics and Applications. ICIRA 2009. Lecture Notes in Computer Science, vol 5928. Springer, Berlin, Heidelberg

[24] J. Ocampo-Jiménez, A. Muñoz-Meléndez, and G. Rodríguez-Gómez, "Enhanced Locomotion of a Spherical Robot Based on the Sea-urchin Characteristics," in Biomimetic and Biohybrid Systems: Third International Conference, Living Machines 2014, Milan, Italy, July 30 -- August 1, 2014. Proceedings, A. Duff, N. F. Lepora, A. Mura, T. J. Prescott, and P. F. M. J. Verschure, Eds. Cham: Springer International Publishing, 2014, pp. 238-248.

[25] B. Wagenknecht and D. Apostolopoulos, "Locomotion strategies and mobility characterization of a spherical multi-legged robot," in Proceedings of the ASME Design Engineering Technical Conference, 2010, vol. 2, no. PARTS A AN.

[26] S. Mahboubi, M. M. S. Fakhrabadi, and A. Ghanbari, "Design and Implementation of a Novel Hybrid Quadruped Spherical Mobile Robot," Robot. Auton. Syst., vol. 61, no. 2, pp. 184-194, 2013.
[27] R. Armour, K. Paskins, A. Bowyer, J. Vincent, and W. Megill, "Jumping robots: a biomimetic solution to locomotion across rough terrain," Bioinspir. Biomim., vol. 2, no. 3, p. S65, 2007.

[28] A. Shiotsu, M. Yamanaka, Y. Matsuyama, H. Nakanishi, Y. Hara, T. Tsuboi, T. Iwade, Y. Sugiyama, and S. Hirai al., "Crawling and Jumping Soft Robot KOHARO," 2005.

[29] M. Artusi, M. Potz, J. Aristizabal, C. Menon, S. Cocuzza, and S. Debei, "Electroactive Elastomeric Actuators for the Implementation of a Deformable Spherical Rover," IEEE/ASME Trans. Mechatronics, vol. 16, no. 1, pp. 50-57, Feb. 2011.

[30] K. W. Wait, P. J. Jackson, and L. S. Smoot, "Self locomotion of a spherical rolling robot using a novel deformable pneumatic method," in 2010 IEEE International Conference on Robotics and Automation, 2010, pp. 3757-3762.

[31] J. Luo, Y. Peng, S. Xie, X. Zou, C. Zhu, K. Feng and J. Gu al., "Modeling of an anti-interference spherical robot for polar region scientific research," 2014 IEEE International Conference on Information and Automation, ICIA 2014. pp. 1300-1305, 2014

[32] S. Zhang, X. Fang, S. Zhou, and K. Du, "Kinetic Model for a Spherical Rolling Robot with Soft Shell in a Beeline Motion," J. Multimed., vol. 9, no. 2, pp. 223-229, 2014. 